\title{
Suprachoroidal Gas: A Rare Complication of Intravitreal Injection of Perfluoropropane
}

\author{
Liana Dedina Wilson Wong Shane Durkin \\ Ophthalmology Unit, The Queen Elizabeth Hospital, Adelaide, SA, Australia
}

\section{Keywords}

Suprachoroidal gas $\cdot$ Complication $\cdot$ Perfluoropropane $\cdot \mathrm{C}_{3} \mathrm{~F}_{8} \cdot$ Intravitreal injection $\cdot$

Pneumatic displacement

\begin{abstract}
This is a case report of a 75-year-old pseudophakic male, who presented with a massive submacular hemorrhage on a background of neovascular age-related macular degeneration. Intravitreal perfluoropropane was used to attempt pneumatic displacement of the submacular hemorrhage. The next day, subconjunctival gas was observed, with no gas seen in the vitreous cavity. Fundal examination showed suprachoroidal detachment. CT images confirmed gas entrapment, with no choroidal hemorrhage identified. The following case report describes suprachoroidal gas as a complication of intravitreal injection of perfluoropropane for pneumatic displacement of submacular hemorrhage. To our knowledge, this is the first such case in the literature. We describe the approach in differentiating suprachoroidal gas from hemorrhage and comment on a plausible mechanism for this complication. This report also serves as a review of the current state of knowledge in the area of suprachoroidal gas as a complication of pneumatic retinopexy and sutureless vitrectomy.
\end{abstract}

\section{Introduction}

The entrapment of gas in the suprachoroidal space as a complication of pneumatic retinopexy was first described by Baker and Hainsworth [1]. Since then, an additional 6 cases were reported in the literature: 2 cases were similarly associated with pneumatic retinopexy [2], 
whilst the others resulted from 23-gauge sutureless pars plana vitrectomy [3-5]. Both perfluoropropane $\left(\mathrm{C}_{3} \mathrm{~F}_{8}\right)$ and sulfur hexafluoride $\left(\mathrm{SF}_{6}\right)$ were implicated [1-6]. Herein, we describe a case of suprachoroidal gas as a complication of intravitreal injection of perfluoropropane for pneumatic displacement of submacular hemorrhage. To our knowledge, this is the first such case appearing in the literature.

\section{Case Report}

A 75-year-old male with a background of bilateral neovascular age-related macular degeneration presented for an unscheduled review 4 weeks after his usual intravitreal injections of bevacizumab. Treat and Extend protocol is used in our clinical setting for managing neovascular age-related macular degeneration. Notable medical history included cervical osteomyelitis after spinal surgery, resulting in fixed flexion at his neck.

The patient was seen 4 weeks before, receiving his usual bilateral intravitreal bevacizumab at a 6-week interval (different batch numbers used). At presentation, his best-corrected visual acuity in the right was $6 / 18$ and the left was $6 / 18$. On presentation, the patient complained of a 6-day history of right eye blurriness, and his visual acuity was drastically reduced to counting fingers (CF). The examination showed a massive submacular hemorrhage (Fig. 1). Bevacizumab was immediately injected in the outpatient setting with the patient lying supine (albeit limited by patient's fixed neck flection deformity) with a plan to proceed to pneumatic displacement of the submacular hemorrhage.

The next day, intravitreal injection of $0.3 \mathrm{~mL}$ of pure $\mathrm{C}_{3} \mathrm{~F}_{8}$ and $50 \mu \mathrm{g} / 0.1 \mathrm{~mL}$ of alteplase was given, followed by anterior chamber paracentesis. "Drinking bird" maneuver was implemented. The patient complained of a postinjection headache, which settled with simple analgesia. On review at day 1 after gas injection, visual acuity in the right eye was hand movements (HM) with a normal intraocular pressure of $11 \mathrm{~mm} \mathrm{Hg}$, clear cornea, and a formed and quiet anterior chamber. Notably, small gas bubbles were observed subconjunctivally in the superior-nasal
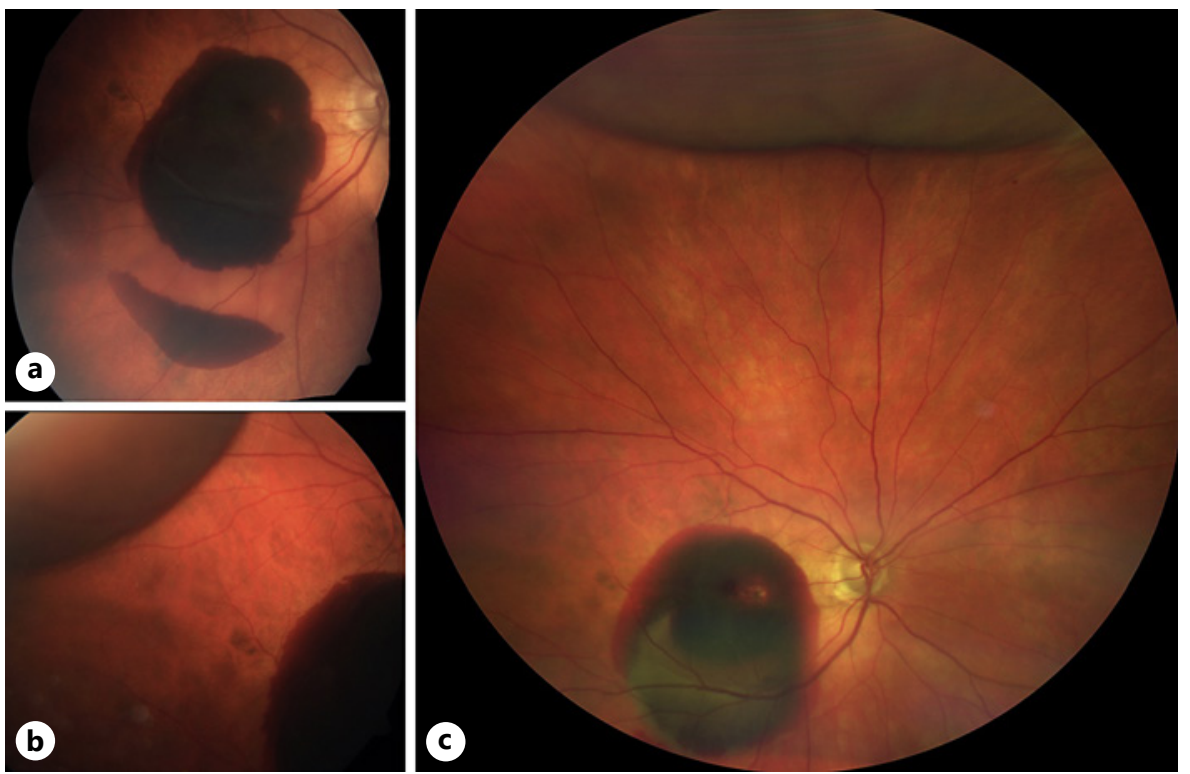

Fig. 1. Color fundus photographs of the right eye illustrating macular hemorrhage at presentation (a), followed by images of suprachoroidal gas taken on day 2 and 3 weeks after injection $(\mathbf{b}, \mathbf{c})$. 

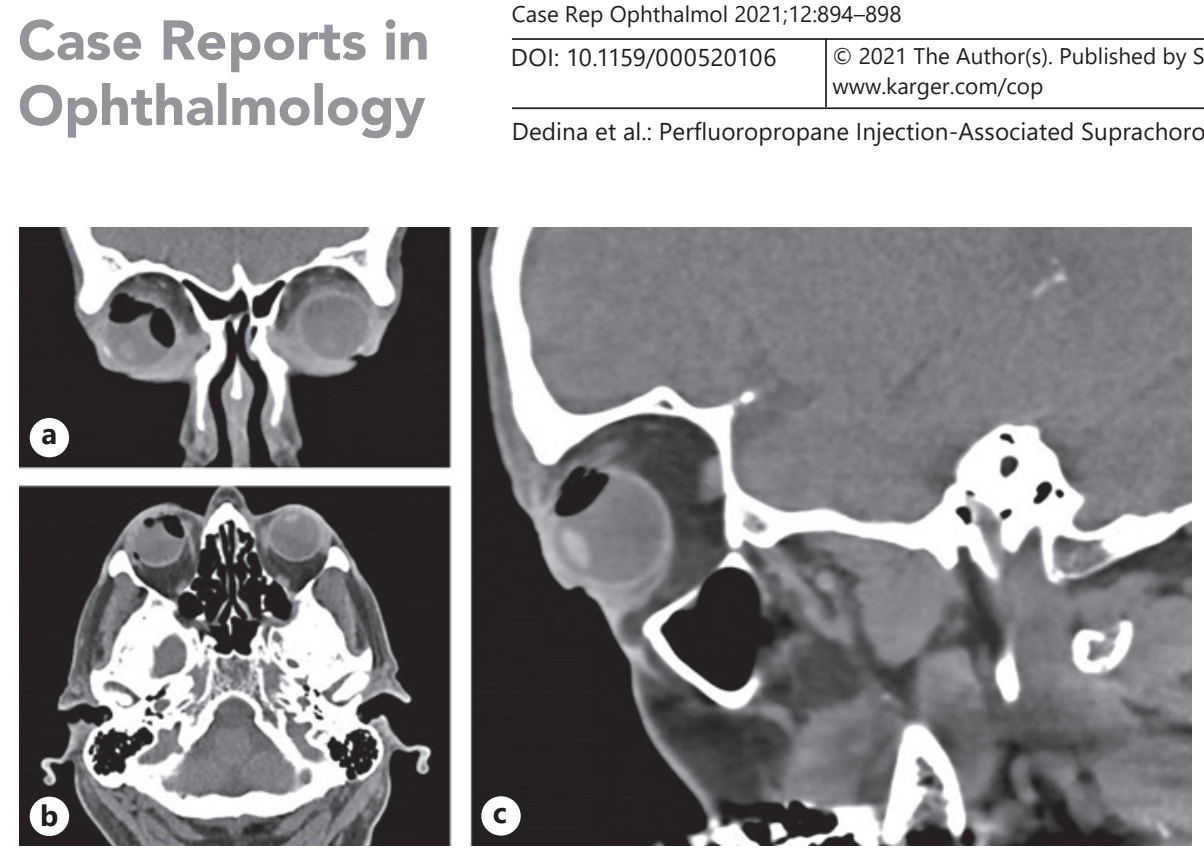

Fig. 2. CT scans showing the presence of suprachoroidal gas in the right eye as seen in different cross-sections: coronal (a), axial (b), and sagittal (c).

quadrant. Fundal examination revealed a suprachoroidal detachment from 11 to 1 o'clock (Fig. 2) with no gas visible in the vitreous cavity.

CT orbits followed urgently to differentiate between an expanding suprachoroidal gas or suprachoroidal hemorrhage. CT imaging confirmed gas locules (Fig. 2). The patient was closely observed with repeated fundal imaging completed at 3 weeks after injection. These show reducing gas (Fig. 1). The suprachoroidal gas resolved fully at 5 weeks after injection.

\section{Discussion/Conclusion}

Iatrogenic suprachoroidal gas was previously described as a complication of both pneumatic retinopexy $[1,2]$ and 23-gauge vitrectomy [3-6]. Pure $\mathrm{C}_{3} \mathrm{~F}_{8}$ and $\mathrm{SF}_{6}$ gases were implicated in these cases [1-6]. Apart from 1 case [3], where $\mathrm{SF}_{6}$-associated choroidal detachment was drained intraoperatively, full spontaneous resolution of gas was observed over days to weeks in other cases. No residual complications were reported. Nevertheless, it is not known if gas entrapment in the suprachoroidal space may result in any long-term neuronal damage or choroidal vascular compromise.

A possible mechanism of iatrogenic suprachoroidal gas after intravitreal injection was suggested by Baker and Hainsworth [1]. It was proposed that excessive withdrawal of the needle tip could result in gas entrapment between sclera and pars plana. In our case, the patient's past neck surgery resulted in a fixed flexion deformity, complicating the injection technique and serving as a possible risk factor. This and other patient-related factors must always be considered prior to injection with an aim to anticipate possible complications. To prevent such complications from occurring, a few extra precautions should be taken: visualization of the needle in the vitreous cavity prior to injection and ensuring the gas is fully injected prior to needle withdrawal. In our practice, we also examine the fundus immediately after injection, to ensure there is perfusion, and the gas bubble can be confidently visualized.

Both ultrasound $[1,2,6]$ and CT [6] imaging modalities were reported as a mode of distinguishing between suprachoroidal gas and hemorrhage. Baker and Hainsworth [1] described the utility of ultrasound, noting that gas totally reflects ultrasound waves. However, this modality may be difficult to interpret the findings in presence of concurrent intravitreal gas, where total reflection of ultrasound waves would prevent visualization of posterior eye structures, 
such as the choroid. It seems that CT imaging may offer a superior approach in distinguishing between gas and hemorrhage, as described by Uji [6]. In our case, CT imaging offered a definitive and prompt visualization of gas, whilst also ruling out a hemorrhagic event. The authors acknowledge that the risks of radiation, comorbid state, and patient's age may influence the selection of the most appropriate imaging modality.

Pain and headache associated with suprachoroidal gas were previously described $[2,6]$. Jabaly-Habib et al. [2] reported 2 cases, where pain persisted until the gas was fully resorbed. In a different case, a mild and unsustained headache was documented [6]. Similar to the latter, our patient complained of a mild headache for a short time after the injection, which fully resolved with simple analgesia. It has been suggested previously that tapping the gas may be indicated in cases of unrelenting and severe pain [2].

Ocular venous air embolism (OVAE) must be acknowledged as a possible, yet highly unlikely, complication of suprachoroidal gas entrapment after intravitreal injection. OVAE has been described as a rare complication of air infusion during vitrectomy; most of these cases are, however, fatal [7]. Here, the mechanism involves pressurized air entering vortex veins through surgical wounds, or alternatively, via a slipped cannula expanding the suprachoroidal space and resulting in damage to vortex veins. The minimal lethal volume of air embolism in humans is not known. However, a rapid infusion of as little as $200 \mathrm{~mL}$ of air resulting in human mortality was reported [8]. Although unlikely, it is nevertheless possible for OVAE to occur in cases such as ours.

Herein, we described a novel case of suprachoroidal gas as a complication of intravitreal injection of $\mathrm{C}_{3} \mathrm{~F}_{8}$ for pneumatic displacement of submacular hemorrhage. To our knowledge, this is the first such case to appear in the literature.

\section{Statement of Ethics}

Written informed consent was obtained from the patient for publication of this case report and accompanying images. The Central Adelaide Local Health Network Human Research Ethics Committee (CALHN HREC) determined the article to be appropriate according to the requirements of the National Health and Medical Research Council (NHMRC) National Statement on Ethical Conduct in Human Research incorporating all updates. This study protocol was reviewed and approved by CALHN HREC, Approval No. 14000, approved on 9/11/2020.

\section{Conflict of Interest Statement}

The authors have no conflicts of interest to declare.

\section{Funding Sources}

No funding was received for publication.

\section{Author Contributions}

Liana Dedina wrote the manuscript. All authors contributed to the conception and design of the work. All authors contributed to drafting and editing of the work. All authors approved the final version of the manuscript as presented above.

\section{Karger'}




\section{Data Availability Statement}

Apart from information presented, no other data are available for publication or sharing.

\section{References}

1 Baker SR, Hainsworth DP. Suprachoroidal gas as a complication of pneumatic retinopexy. Retina. 2000;20(2): 224-5.

2 Jabaly-Habib HY, Fineberg EM, Tornambe PE, Lang YZ, Garzozi HJ. Prolonged pain following unintentional injection of gas into the suprachoroidal space during pneumatic retinopexy. Retina. 2003;23(5):722-3.

3 Tarantola RM, Folk JC, Shah SS, Boldt HC, Abràmoff MD, Russell SR, et al. Intraoperative choroidal detachment during 23-gauge vitrectomy. Retina. 2011;31(5):893-901.

4 Lin CJ, Peng KL. Intraoperative severe suprachoroidal air as a complication of 23-gauge vitrectomy combined with air-fluid exchange. Int Med Case Rep J. 2018;11:173-6.

5 Saxena RK, Rani PK. Suprachoroidal air during silicone oil removal. BMJ Case Rep. 2019;12(5):e230207.

6 Uji A. Suprachoroidal gas injection as a complication of pars plana vitrectomy confirmed by computed tomography. Clin Ophthalmol. 2012;6:533-6.

7 Morris RE, Boyd GL, Sapp MR, Oltmanns MH, Kuhn F, Albin MS. Ocular venous air embolism: a report of 5 cases. J Vitreoretin Dis. 2019;3(2):107-10.

8 Toung TJ, Rossberg MI, Hutchins GM. Volume of air in a lethal venous air embolism. Anesthesiology. 2001; 94(2):360-1. 